\title{
Un simple outil de surveillance ? Biographie d'une caméra portée sur le corps par la police aux Pays-Bas ${ }^{1}$
}

\section{A simple surveillance tool? The story of a body camera worn by the police in the Netherlands}

\author{
Lucie Dalibert ${ }^{1}$, Tjerk Timan $^{2}$ \\ ${ }^{1}$ S2HEP, Université Claude Bernard Lyon 1, France, lucie.dalibert@univ-lyon1.fr \\ ${ }^{2}$ TNO, La Hague, Pays-Bas, tjerk.timan@tno.nl
}

\begin{abstract}
RÉSUMÉ. Cet article construit la biographie d'un objet technologique récent et non stabilisé : un type de caméra portée par la police aux Pays-Bas appelé Zepcam. En examinant les différentes inscriptions et traductions auxquelles sa conception et son introduction ont donné lieu, il retrace dans quelle mesure les finalités, les actions et finalement la signification de cet objet technologique se forment et se transforment au contact des différents acteurs qui s'en saisissent : responsables politiques, concepteurs et utilisateurs. Ce faisant, il participe au façonnement d'une culture technique, indispensable pour comprendre le sens des objets technologiques.

ABSTRACT. This article presents the story of a recent and unstabilized technological object: Zepcam - a type of body camera, worn by the police in the Netherlands. By examining the various inscriptions and translations that took place during its conception and introduction, the paper traces the extent to which the objectives, actions and ultimately the meaning of this technological object are formed and transformed when coming into contact with the various actors who appropriate it: policy-makers, designers and users. In doing so, it contributes to the shaping of a technical culture, which is essential for understanding the meaning of technological objects.

MOTS-CLÉS. Biographie, caméra portée sur le corps, police, script, conception, usages, surveillance.

KEYWORDS. Story, body camera, police, script, conception, uses, surveillance.
\end{abstract}

En 2009, suite à des résultats jugés positifs au Royaume-Uni, les Pays-Bas ont commencé à tester et déployer les premières caméras portées sur le corps (bodycameras) par la police. Contrairement aux États-Unis où ces appareils ont fait l'objet de débats et de controverses quant à leur objectif et leur utilisation, leur introduction s'est faite de façon presque silencieuse aux Pays-Bas. Dans les pays anglo-saxons, mais aussi en France, ces caméras portées ont été présentées comme un témoin objectif des actes de la police, donc comme ayant le potentiel de contrôler et discipliner les actions des policiers. Elles permettraient ainsi d'agir sur l'image publique de la police [SCH 15]. Si l'introduction des caméras portées a donné lieu à des discussions sur la légitimité et la transparence (des pratiques) de la police [TYL 04], elle a aussi soulevé des craintes et des interrogations quant à la surveillance accrue des citoyens [LIP 16]. Alors qu'il est prévu d'intégrer ces caméras à l'équipement standard de la police néerlandaise, elles n'ont pas fait l'objet de telles discussions aux Pays-Bas. Peu se sont également penchés sur ces objets particuliers pour interroger pourquoi et comment ils ont été conçus [LIP 16]. Ainsi, cet article entend retracer la biographie d'un type de caméra portée, la Zepcam, en tant qu'outil de surveillance de la vie nocturne, lequel a fait l'objet d'une phase de test aux Pays-Bas de 2009 à 2012.

En analysant les considérations qui ont présidé au développement de la caméra portée sur le corps par la police, les décisions et traductions qui ont eu lieu lors de sa conception, ainsi que son usabilité et ce que les usagers font à la caméra, cet article participe au façonnement d'une culture technique, indispensable pour comprendre les objets technologiques. Il participe aussi à la délimitation même de

${ }^{1}$ Certaines parties de cet article ont été publiées dans le chapitre [TIM 17]. 
cette notion. Avec la notion de culture technique, Chouteau et ses collègues invitent à appréhender les objets technologiques à trois niveaux : celui de la connaissance de leur fonctionnement (la perspective de l'ingénieur), celui des savoir-faire développés pour les manier (la perspective de l'usager), et celui des savoirs sur leur mode d'existence permettant de penser leur sens (la perspective du citoyen) [CHO 17]. Comme nous le montrerons, la signification de l'objet technologique n'est pas fixe mais se forme et se transforme au contact des différents acteurs qui s'en saisissent et ne constitue pas, en soi, une perspective à part.

D'abord, nous présenterons le cadre conceptuel que nous mobilisons pour appréhender la Zepcam. Ensuite, après avoir exposé notre étude de cas et notre méthodologie, nous nous intéresserons à la façon dont les responsables politiques, se voulant concepteurs, «inscrivent» les designers, puis à la façon dont les utilisateurs-policiers sont également configurés par les concepteurs. Un objet technologique visant à être utilisé, sa biographie ne saurait s'arrêter à la phase de conception : nous nous tournerons, enfin, vers les pratiques d'utilisation de cette caméra, lesquelles résistent au script initial et donnent lieu à de nouveaux usages.

\section{Script et biographie comme rétro-ingénierie des caméras portées}

Comme l'ont montré de nombreux travaux ancrés dans les études sur les sciences et les technologies, les objets technologiques sont non seulement le résultat d'une construction sociale [PIN 12], mais concevoir et fabriquer de tels objets implique également d'envisager et de construire des mondes sociaux particuliers [BIJ 92]. Si l'approche dite de la «construction sociale des technologies » a souligné l'importance de facteurs non-techniques dans le développement des objets technologiques et mis en évidence que, pour ces derniers, « les choses auraient pu être différentes » [PIN 12], d'autres se sont davantage tournées vers les objets eux-mêmes, notamment leurs actions et les réseaux dans lesquels ils sont inscrits.

La sociologie de la traduction a mis l'accent sur le fait que les objets technologiques ne sont pas des entités passives mais qu'ils sont dotés de capacité d'action (agency), affectant le réseau d'objets et d'êtres humains auquel ils sont liés [AKR 90] [AKR 92]. Au sein de ce courant, Akrich a proposé un cadre d'analyse particulièrement riche pour comprendre le processus de conception d'un objet technologique et, ce faisant, construire sa biographie. Elle a montré que pendant la phase de conception, les ingénieurs anticipent les motivations, les compétences et les comportements des futurs utilisateurs. Pour en rendre compte, elle a proposé la notion de «script», qui invite à appréhender l'objet technologique de façon analogue à un scénario de film, celui-ci « définissant un espace, des rôles et des règles d'interaction entre les différents acteurs (humains et non-humains) qui viendront incarner ces rôles » [AKR 90, p. 85]. Le «script» attire l'attention sur le fait que les concepteurs inscrivent dans l'objet technologique un usage(r) imaginé, orientant ainsi l'interaction entre l'utilisateur et l'objet [AKR 92]. En d'autres termes, ils «configurent» les utilisateurs ${ }^{2}$ [WOO 90]. Ainsi, les objets technologiques attribuent et délèguent des compétences, des actions et des responsabilités spécifiques aux utilisateurs et aux objets technologiques, ces derniers pouvant créer de nouvelles « géographies de responsabilités » et transformer ou renforcer celles qui existent déjà [AKR 92]. En résumé, l'analyse de script permet de comprendre comment les concepteurs envisagent le monde dans lequel l'objet technologique va s'insérer et, ce faisant, inscrivent cette vision dans l'objet à développer.

\footnotetext{
${ }^{2}$ Akrich a montré que l'utilisateur envisagé tend à être un usager, non une usagère [AKR 95]. Supposant que leurs préférences et compétences sont représentatives de celles des utilisateurs, les concepteurs se projettent comme les utilisateurs potentiels : leurs préférences sont matérisalisées dans l'objet technologique et son script. Oudshoorn et ses collègues ajoutent, qu'en pratique, configurer l'utilisateur revient souvent à concevoir un produit à destination des hommes jeunes, blancs, avec un haut niveau d'éducation et de compétences techniques : un utilisateur qui reflète la composition du groupe de concepteurs [OUD 04].
} 
Dans le cas de la caméra portée sur le corps, les concepteurs ne sont pas les seuls qui ont inscrit une certaine vision des fonctions et de l'utilisation de l'objet dans celui-ci. D'autres parties prenantes ont influencé la nature de cet appareil en amont du processus de conception. Si les concepteurs « inscrivent » l'utilisateur final, ils ont eux-mêmes été contraints voire «configurés » par d'autres : les responsables politiques ont défini des prérequis et des objectifs spécifiques pour cette caméra. Par ailleurs, pour comprendre comment un tel objet technologique "se comporte", nous devons non seulement analyser comment cette caméra a été conçue mais aussi considérer les pratiques d'utilisation par la police, lesquelles transforment les actions et le sens de cet objet technologique.

\section{2. Étude de cas et méthodologie}

La biographie proposée ici est construite à partir de l'analyse du script d'un type de caméra portée, la Zepcam (figure 1). Celle-ci a été conçue en 2009 par une entreprise du même nom basée à Amsterdam, Pays-Bas. Alors que cette entreprise fabriquait des caméras haut de gamme pour sports extrêmes, à l'issue d'un appel d'offres le gouvernement néerlandais lui a demandé de développer sa technologie pour un marché différent, celui de la sécurité.

La Zepcam est composée de trois parties : (1) l'objectif (la caméra) qui est connecté au (2) boîtier de batterie et d'interface-utilisateur, et (3) un bracelet de télécommande.

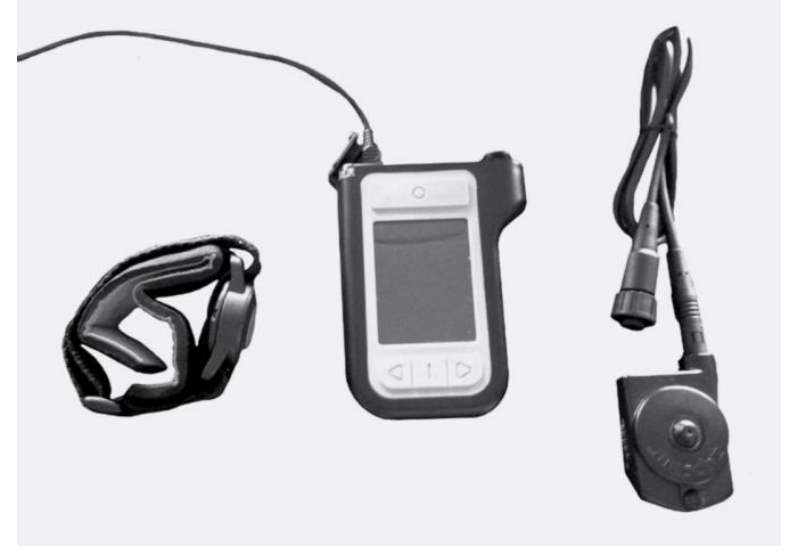

Figure 1. La Zepcam

De gauche à droite : le bracelet, le boîtier d'interface et la caméra

Comme nous l'a expliqué le fondateur et président-directeur général (PDG) de Zepcam, plusieurs accessoires viennent compléter la caméra portée : il s'agit de clips permettant de la positionner sur différentes parties du corps (torse, épaule ou casque) et dont l'utilisation dépend fortement (entretien, PDG de Zepcam, 3 mars 2012). La Zepcam peut enregistrer jusqu'à 4 heures consécutives de séquences vidéo, en mode vision de jour ou vision de nuit. Elle peut être configurée pour enregistrer des séquences audiovisuelles ou uniquement visuelles. Les images peuvent être visionnées et étiquetées (tagged) directement sur la caméra et une fois téléchargées.

Afin de construire sa biographie, nous nous appuyons sur :

-(1) les entretiens réalisés par le second auteur avec le PDG de Zepcam (3 mars 2012), les responsables politiques (Taskforce GTPA Rotterdam le 11 septembre 2012 et police HollandMidden le 2 octobre 2012) et les officiers de police impliqués dans le développement et la première expérience d'utilisation de la caméra portée sur le corps aux Pays-Bas (entretiens réalisés à Rotterdam le $1^{\mathrm{e}}$ décembre 2012 [policiers 1 et 2] et Enschede le 11 septembre 2012 [policier 3]),

-(2) deux séries d'observations participantes nocturnes effectuées avec des policiers équipés de Zepcam (Enschede, 3 octobre 2012 de $22 \mathrm{~h}$ à $6 \mathrm{~h}$ et 10 novembre 2012 de $22 \mathrm{~h}$ à $3 \mathrm{~h}$ ), 
-(3) l'analyse des documents-cadre circulant au sein des forces de police néerlandaises concernant l'utilisation de la caméra portée [HOM 03] [JAN 09] [HAM 10],

-(4) la manipulation par les auteurs d'une caméra prêtée par Zepcam.

C'est grâce aux contacts obtenus lors de recherches antérieures que nous avons eu accès aux différents sites et c'est par «boule de neige » que le réseau relativement restreint de personnes impliquées dans la conception et l'utilisation des caméras portées aux Pays-Bas a été cartographié et contacté. Nous avons analysé le matériel collecté avec le logiciel d'analyse TAMS; nos schémas de codage ont été développés sur la base des items préparés pour nos entretiens semi-structurés.

\section{Le souhait des responsables politiques d'être à l'origine d'une innovation}

Les premières caméras portées sur le corps par la police ont été développées par Zepcam à la demande du gouvernement néerlandais. Ces caméras, dont la mobilité introduit dans l'espace public un type nouveau de caméra de surveillance, ont été largement influencées par la volonté des responsables politiques d'être à l'origine d'une innovation technologique, voire d'être eux-mêmes des innovateurs.

\subsection{Définition des fonctions des caméras portées}

Les visées des caméras portées ainsi que « la géographie des responsabilités » [AKR 92] — la répartition des charges et obligations - envisagée entre les différents acteurs concernés par l'utilisation de cet objet technologique sont abordées dans plusieurs rapports-cadre [HOM 03] [JAN 09] [FER 10]. Dans l'un d'eux, lequel précède le développement de la caméra portée, cinq objectifs sont mentionnés [JAN 09]. La caméra vise à :

(1) enregistrer et réduire les actes de violence à l'encontre de la police ;

(2) enregistrer des infractions et identifier les suspects ;

(3) enregistrer les troubles à l'ordre public ;

(4) promouvoir un sentiment de sécurité pour la police ;

(5) utiliser les images filmées comme preuves à l'appui dans les enquêtes criminelles.

La priorité est donnée à la sécurité des agents de police (objectifs 1 et 4). Les caméras portées sur le corps ne sont pas tant pensées comme des «caméras de vidéosurveillance mobiles » qui peuvent et doivent surveiller les espaces publics dans lesquels elles sont déployées, que comme des aides et des outils de protection pour les policiers.

C'est ce que confirme l'un des responsables impliqués dans le projet pilote d'introduction de ces caméras au sein de la police à Rotterdam :

« R : Rotterdam a rejoint l'un des pilotes pour que ces caméras soient également testées à Rotterdam, l'une des principales forces de police du pays, où il y a beaucoup de violences contre les policiers, afin de réduire ces violences.

A : La réduction de la violence était la raison principale pour laquelle la caméra devait être utilisée?

$\mathrm{R}$ : Pour nous ça l'était, oui » (Responsable Rotterdam).

Cependant, si la finalité principale de la caméra portée est la protection des agents de police, d'autres objectifs et utilisations, qui peuvent être en tension les uns avec les autres, sont dors et déjà envisagés, à savoir la collecte de preuves, la recherche de potentielles infractions et l'identification de suspects. Ce phénomène d'élargissement (et d'éventuel détournement) des fonctions initiales (function creep) d'une technologie est bien connu des études sur la surveillance. Lyon le définit comme le 
processus dans lequel «des usages ultérieurs nouveaux sont imaginés pour des systèmes techniques existants et sont ajoutés à la panoplie de fonctions d'origine » [LYO 05, p. 67, notre traduction]. Il fait référence au fait qu'une fois qu'une technologie a été développée et intégrée au tissu social, les utilisateurs, les concepteurs et/ou les parties prenantes lui «découvrent» des fonctionnalités additionnelles et les déploient, comme lorsque les radars qui servent au départ à contrôler la vitesse des automobilistes sont utilisés pour reconnaître les plaques d'immatriculation et suivre la trace des véhicules suspects, ou lorsque qu'une caméra portée sur le corps par la police, à l'origine conçue pour protéger cette dernière contre des actes de violence, peut être utilisée pour enregistrer et identifier les citoyens.

Ainsi, comme l'indique le responsable politique cité plus haut:

«Si vous utilisez la caméra, alors vous constatez qu'elle contribue, ou au moins qu'elle est utile pour capturer des images qui peuvent vous aider plus tard dans la recherche d'incidents et de leurs responsables » (Responsable Rotterdam).

Si à l'origine du développement des caméras portées par la police se trouvent les responsables politiques et le problème principal qu'ils ont posé - la violence à l'encontre de la police et le besoin de protection des agents - les objectifs que l'objet technologique est censé remplir sont toutefois plus divers. La caméra doit certes permettre un sentiment de sécurité pour la police mais aussi l'enregistrement des infractions et troubles à l'ordre public, l'identification de leurs suspects et l'utilisation de ces images comme pièces à conviction. Ces objectifs constituent autant de lignes directrices, et donc non seulement des fins mais aussi des contraintes, que les concepteurs devront respecter et traduire dans la construction de la caméra portée.

\subsection{Inaltérabilité et sécurité des images}

Dans ce cahier des charges défini par les responsables politiques, la question des images a pris une place particulière. Ce faisant, ce n'est plus l'objectif de sécurité des agents de police qui tend à primer mais ceux ayant trait à la collecte de preuves et l'identification des suspects, donc à la surveillance des citoyens.

En effet, dans les documents-cadre, les responsables politiques posent certaines contraintes quant aux fonctions et à l'utilisation des caméras portées :

«L'enregistrement par caméra [portée] doit avoir lieu dans l'espace public. Ces enregistrements ne peuvent être effectués que par des agents de police [...]. Pour ce type d'enregistrement par caméra, il n'existe aucune autre base légale que celle mentionnée à propos des enquêteurs agissant en vertu de l'article 2 de la loi sur la police. [...] Il est à noter que, si possible, l'agent indique toujours clairement qu'il enregistre une vidéo. Le stockage des images et son traitement relèvent du champ d'application des articles 8 et 9 de la loi sur la protection des données de police [...] À l'intérieur de la police, les images sont stockées sur une machine dite autonome, sans connexion Internet, dans une pièce sécurisée et verrouillée du poste de police » [JAN 09].

Les images filmées par les caméras portées sont régies par la loi sur la protection des données de la police ${ }^{3}$, laquelle stipule les droits et obligations liés aux données personnelles collectées par la police. Dès lors, les séquences filmées par les caméras portées ne relèvent pas de la même législation que les caméras de vidéosurveillance. Cela s'explique par le fait que ces dernières surveillent les espaces

\footnotetext{
${ }^{3}$ Le texte de loi est accessible ici : https://wetten.overheid.nl/BWBR0022463/2016-01-01 ; depuis mai 2018, cette loi est remplacée par la Directive européenne de police : https://eur-lex.europa.eu/eli/dir/2016/680/oj (consultés le 23 mai 2019).
} 
publics sur de longues périodes, tandis que les caméras portées ne peuvent être utilisées que lorsque l'utilisateur (l'agent de police) décide qu'une situation spécifique doit être filmée. Il lui appartient donc de déterminer ce qui vaut la peine d'être enregistré : cette responsabilité lui est déléguée. Cela signifie que les caméras portées par la police, lesquelles produisent des images sensibles, ne constituent pas un outil de surveillance régulière, mais qu'elles sont plutôt à la fois un instrument de protection des agents de police et un instrument de collecte de preuves (y compris pour l'identification des suspects). Ceci est particulièrement manifeste dans la prescription concernant la conservation des images : pour garantir leur sécurité et inaltérabilité, le document-cadre stipule que les séquences filmées par la caméra doivent être stockées localement, sur un appareil qui n'est connecté à aucun réseau.

Cet impératif s'ajoute aux objectifs définis par les responsables politiques concernant ce que la caméra doit permettre d'accomplir. Outre que cette prescription tend à modifier la hiérarchie des objectifs - d'un outil pour la protection des policiers, la caméra portée devient principalement un outil de collecte de pièces à conviction - elle s'ajoute au cahier des charges qui fixe les prérequis et contraintes avec lesquels devront faire les concepteurs. Comme l'indique l'un des responsables politiques au sujet de la véracité des images :

«A: $[\ldots]$ et lorsqu'il est question de recherche de la vérité $[\ldots]$, peut-on faire confiance aux images des caméras portées?

$\mathrm{R}$ : Oui. C'est pourquoi le temps d'enregistrement de la vidéo doit être long. La date doit être visible afin qu'il ne puisse y avoir aucune coupe ou édition. Pour toutes les séquences images, un horodatage doit être visible. Nous en avons explicitement tenu compte car nous ne voulions pas créer de possibilité de copié-collé dans nos images. Si nous capturons des images, il faut que ce soit les [vraies] images. [...] La raison qui conduit à un incident, ce qui se passe exactement jusqu'à l'arrestation... et peut-être aussi le transport du suspect, tout cela devrait faire partie de la séquence image » (Responsable Rotterdam).

En termes de script, cela signifie que les responsables politiques veulent un dispositif qui aide les policiers en service tout en fournissant des séquences vidéo inaltérées et inaltérables. Reste toutefois aux concepteurs de déterminer comment cette exigence doit être implémentée dans la caméra.

\subsection{Visibilité de la caméra et délégation de responsabilités}

À cet impératif est liée une autre demande : dans quelle mesure les personnes surveillées devraientelles être informées et assurées de la «justesse» de la caméra et des images enregistrées ? Le document-cadre susmentionné fournit un script concernant la visibilité de la caméra pour le sujet filmé :

«[S]i possible, l'agent indique toujours clairement qu'il enregistre une vidéo. [...] Il doit être exprimé clairement et sans ambiguïté à tous ceux qui participent à un incident ou à une conversation qu'une caméra est utilisée. Cela peut se faire via une communication verbale ou un badge avec une inscription clairement visible pour tous » [JAN 09].

Lorsque la caméra portée est utilisée pour filmer une situation, cette utilisation ne saurait être dissimulée. Au contraire, sa présence et son action doivent être rendues visibles ou être communiquées aux personnes filmées. Ici, c'est à l'utilisateur que les responsables politiques délèguent cette responsabilité : c'est aux policiers-utilisateurs, et non aux concepteurs, que revient le devoir d'indiquer, soit oralement soit par le port d'un badge, qu'une caméra est présente et qu'elle est en train de filmer. Cependant, dans les rapports des responsables, ce qui est entendu par visibilité de la caméra ou ce qui constituerait une visibilité suffisante n'est pas spécifié.

Le script élaboré par les responsables repose sur différentes bases juridiques et des documents semiofficiels qui établissent un éventail relativement large et ouvert d'objectifs pour la caméra portée. Celle-ci doit permettre d'enregistrer et réduire les actes de violences à l'encontre de la police et 
promouvoir un sentiment de sécurité pour ses agents, elle doit permettre d'enregistrer les troubles à l'ordre public et les infractions et d'en identifier les suspects, elle doit aussi rendre possible l'utilisation des images filmées comme preuves dans les enquêtes de la police. Aussi, elle ne peut être utilisée que dans la sphère publique, elle doit être visible et les images doivent être stockées dans un dispositif informatique qui n'est pas en réseau afin qu'elles soient sécurisées et inaltérables. Toutefois, l'interprétation de ces finalités est laissée aux concepteurs. Si les responsables se veulent être à l'origine de la caméra portée - et se veulent, en quelque sorte, innovateurs - en l'absence de directives précises, une myriade de choix et de responsabilités est déléguée aux concepteurs et utilisateurs quant à l'élaboration et l'usage de cette dernière, les concepteurs devant en particulier traduire le «script» élaboré par les responsables politiques en des fonctionnalités spécifiques et implémentables dans la caméra.

\section{Des prérequis des responsables politiques à leur traduction par les concepteurs}

Sur la base des directives énoncées par les responsables politiques, les concepteurs ont dû élaborer une caméra portée sur le corps, donc convertir le script et les objectifs initiaux en un objet doté de fonctionnalités. Dans ce processus, des traductions ont été opérées tandis que certains types d'actions ont été déléguées aux utilisateurs et d'autres à la caméra.

\subsection{Des objectifs traduits en lignes directrices}

Lors de la première étape de conception, Zepcam a transformé la gamme de documents politiques hétérogènes en «lignes directrices de conception » auxquelles l'objet devait adhérer. La caméra portée :

(1) « doit être fiable et ouverte,

(2) ne doit pas déclencher de réponses négatives,

(3) doit fournir une bonne qualité d'image (70\% de son utilisation est la nuit),

(4) doit pouvoir agir en tant que « témoin objectif »,

(5) doit permettre différents niveaux d'accès aux images,

(6) peut être utilisée comme outil de formation,

(7) doit recueillir des preuves, ou au moins permettre de rendre compte d'un incident de façon plus simple et fiable qu'un rapport écrit » (PDG de Zepcam).

Lorsque l'on compare ces directives aux demandes formulées par les responsables politiques, c'est une interprétation plus ancrée dans la pratique et centrée sur les utilisateurs que l'on obtient.

Faire en sorte que la caméra ne déclenche pas de réactions négatives est la traduction de l'objectif de protection ou de diminution de la violence à l'encontre des policiers, soit le premier objectif fixé par les responsables. Concrètement, cela demande que le port de la caméra ne présente pas de risque ou ne constitue pas un danger pour l'agent une fois dans l'espace public. Quant à la demande faite par les responsables que la caméra enregistre différentes situations et incidents (actes de violence, troubles à l'ordre public, infractions) et en recueille des preuves, elle est certes traduite en l'exigence que la caméra permette de rendre compte d'un incident de façon simple mais aussi en la nécessité pratique qu'elle soit fiable et qu'elle fournisse une bonne qualité d'image. Concevoir une caméra fiable signifie que la caméra fonctionne toujours lorsque l'on en a besoin : elle doit résister aux conditions extérieures (à l'obscurité et la pluie) et aux actions et gestes des agents de police en intervention (aux chocs et mouvements rapides par exemple). En outre, dans la mesure où l'usage de la caméra portée a principalement lieu la nuit, la qualité de l'image doit être suffisamment élevée pour que les séquences filmées puissent être utilisées pour l'identification ou la reconnaissance faciale des suspects. Ce qui est entendu exactement par une caméra « ouverte » n'est pas clairement défini. Cette caractéristique invite cependant à interroger ce qui devrait être ouvert et pour qui (nous y reviendrons). Quant aux lignes directrices visant à ce que la caméra puisse agir en tant que «témoin objectif » et qu'elle permette 
différents niveaux d'accès aux images, elles sont la traduction de l'objectif fixé par les responsables politiques que les images enregistrées puissent servir de pièces à conviction, ce qui demande qu'elles puissent être vues par d'autres que seul l'agent ayant filmé la scène.

C'est en ces termes ou en ces considérations plus pratiques que les objectifs et demandes énoncés par les responsables politiques ont été traduits et ont pris forme pour les concepteurs. Toutefois, pour fabriquer la caméra portée par la police, Zepcam n'a pas fait le choix de concevoir une nouvelle technologie. Plutôt, l'entreprise s'est appuyée sur un modèle de caméra à destination des sportifs qu'elle commercialisait déjà. Or, utiliser un produit existant et l'adapter aux objectifs fixés par les responsables politiques pour qu'il soit utilisé par la police n'est pas sans contraindre ses fonctionnalités et usages.

\subsection{Matérialiser la transparence de l'appareil, inscrire une géographie de responsabilités}

C'est parce qu'elle produisait des caméras robustes - cf. enregistrement de sports « extrêmes »que l'État néerlandais a choisi Zepcam pour développer des caméras pour la police. Les éléments de base de la caméra - l'objectif, le boîtier d'interface et la télécommande - ont été conservés : résistante aux conditions extérieures, aux chocs et aux mouvements rapides et fournissant une qualité d'image suffisante, celle-ci pouvait convenir aux circonstances d'utilisation de la police. Les lignes directrices visant à ce que la caméra soit ouverte et autorise différents niveaux d'accès ont, quant à elles, donné lieu à des choix de conception spécifiques.

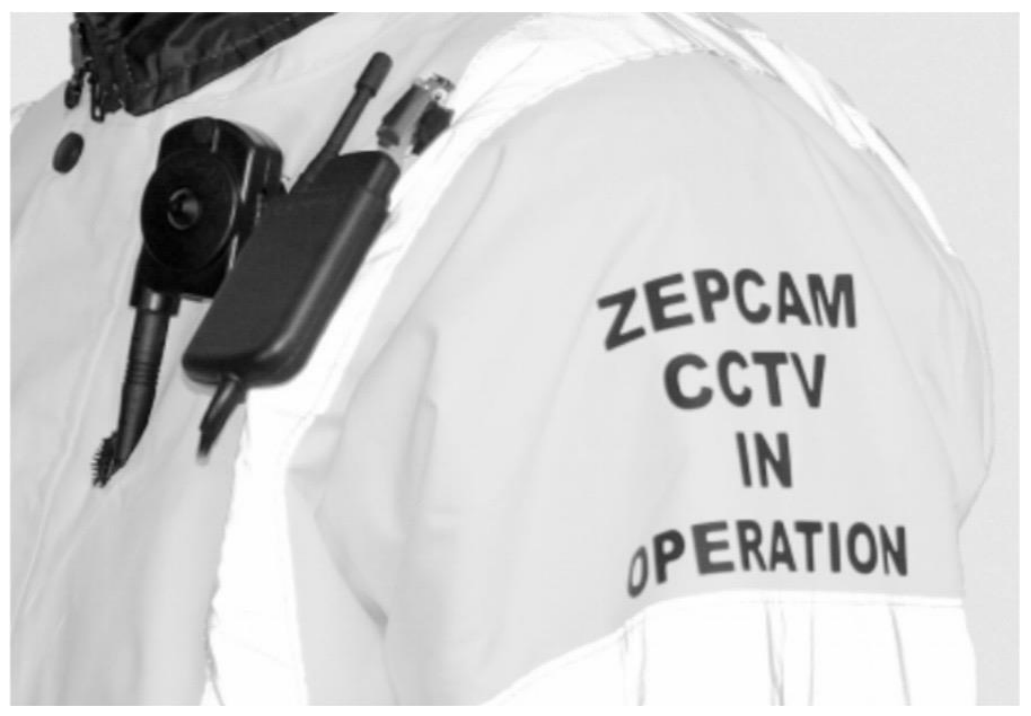

Figure 2. Notification de la présence de la Zepcam

Ce modèle est destiné au marché britannique. Aux Pays-Bas, un badge est porté sur l'uniforme

Selon les lignes directrices de conception définies par Zepcam sur la base des demandes des responsables politiques, la caméra portée doit être un appareil «ouvert». Une des façons de remplir ce prérequis est de s'assurer que le public sait non seulement qu'une caméra est portée mais aussi quand elle enregistre. De façon analogue aux panneaux situés dans les zones urbaines qui avertissent le public qu'il est filmé par des caméras de vidéosurveillance, Zepcam a matérialisé la première condition par un badge que l'on vient attacher à l'uniforme ou par une notification intégrée à celui-ci (figure 2).

La notification de la présence et de l'enregistrement de la caméra portée est également déléguée à une LED clignotante positionnée sur le bracelet, laquelle rappelle la lumière rouge des caméscopes. L'objectif de la caméra, quant à lui, ne fournit aucun retour (feedback). De même, le bracelet, qui tend à être recouvert par les manches de veste de l'agent de police, offre à ce dernier l'occasion d'enregistrer des images secrètement, c'est-à-dire sans que le sujet surveillé ne soit au courant. Cette possibilité de réaliser des enregistrements de manière non transparente peut être considérée comme contraire aux exigences politiques et légales. En fait, l'agent de police est considéré et configuré 
comme le principal, voire le seul, utilisateur. Autrement dit, les concepteurs ont ignoré les «acteurs impliqués » [CLA 93], à savoir les sujets observés et les potentiels enjeux qui découlent de leur présence, comme ceux liés à la surveillance et au respect de la vie privée par exemple.

Outre sa traduction en la visibilité de la caméra (par la notification de sa présence et de son utilisation), l'exigence d'ouverture a conduit Zepcam à développer un logiciel de traitement des séquences vidéos. C'est dans ce logiciel qu'a été adressée et concrétisée la ligne directrice concernant les différents niveaux d'accès aux images, c'est-à-dire qui peut faire quoi avec les images. Tel que l'ont interprété les concepteurs, le contrôle d'accès répond à la demande politique de permettre à la caméra d'être un «collecteur de preuves », fonction pour laquelle les séquences vidéo doivent être inaltérées et inaltérables. Afin de pouvoir utiliser ces séquences à des fins de preuve, l'interface de la caméra autorise l'étiquetage (tag) ou le marquage des images. Cela signifie que depuis le boîtier d'interface, l'utilisateur peut récupérer et sélectionner une partie des séquences vidéo qui ont été filmées et leur attribuer un nom (étiquette). Après que les données ont été téléchargées sur un ordinateur, un utilisateur désigné, disposant d'une autorisation d'accès, peut rechercher cette étiquette au lieu de visionner l'intégralité du film d'une soirée. Toutefois, si l'agent en service peut étiqueter les séquences, une fois qu'elles sont enregistrées, il ne peut ni les supprimer ni les modifier. De même, pour garantir l'intégrité des images, l'écran du boîtier d'interface se verrouille automatiquement au bout d'un temps programmé (environ 5 minutes). Pour le réactiver, l'utilisateur doit saisir le code qui le lie à l'appareil. Enfin, lorsque l'appareil est de retour au poste de police, seul un administrateur local peut accéder aux séquences et éventuellement les supprimer. Autrement dit, le traitement des séquences relève de la responsabilité des services informatiques locaux et/ou des superviseurs (tableau 1). Par ces contrôles d'accès intégrés au logiciel, la fonction «témoin objectif » de la caméra est inscrite dans l'objet technologique.

\begin{tabular}{|c|c|c|c|c|c|}
\hline & \multicolumn{5}{|c|}{ Acteurs } \\
\hline & & $\begin{array}{l}\text { Caméra } \\
\text { portée }\end{array}$ & $\begin{array}{l}\text { Agent de } \\
\text { police }\end{array}$ & $\begin{array}{l}\text { Administrateur } \\
\text { réseau local }\end{array}$ & Superviseur \\
\hline \multirow{7}{*}{ Fonctions } & Enregistrement & $\mathrm{x}$ & $\mathrm{x}$ & & \\
\hline & Étiquetage (tag) & & $\mathrm{x}$ & & \\
\hline & Téléchargement & & & $\mathrm{x}$ & $\mathrm{x}$ \\
\hline & Suppression & $\mathrm{x}$ & & $\mathrm{x}$ & $\mathrm{x}$ \\
\hline & Vérification & & $\mathrm{x}$ & & $\mathrm{x}$ \\
\hline & $\begin{array}{l}\text { Assigner des } \\
\text { utilisateurs }\end{array}$ & & & $\mathrm{x}$ & \\
\hline & $\begin{array}{c}\text { Modification des } \\
\text { paramètres }\end{array}$ & & & $\mathrm{x}$ & \\
\hline
\end{tabular}

Tableau 1. Attribution des responsabilités dans la Zepcam

Les séquences vidéo ont également une durée de vie limitée, la caméra étant dotée d'une fonction d'écrasement (overwrite) : après un nombre d'heures donné, les séquences sont automatiquement 
supprimées sans intervention de l'utilisateur ou de l'administrateur. Les concepteurs ont ainsi inscrit dans l'interface une responsabilité partagée entre le policier, l'administrateur local et l'objet. La décision de créer et de conserver des séquences relève de la responsabilité de l'utilisateur, le bracelettélécommande et l'étiquetage des séquences permettant cette action. Le traitement des séquences est, lui, délégué partiellement à l'appareil et partiellement à l'administrateur local du poste de police. Quant à la confidentialité des personnes filmées, elle est protégée par la fonction de verrouillage automatique, ce qui signifie que si la caméra est perdue ou volée, ses données ne sont pas accessibles.

Ces différentes caractéristiques et fonctionnalités montrent dans quelle mesure les concepteurs ont inscrit, dans l'objet lui-même, les exigences politiques relatives aux caméras portées, et ce en traduisant d'abord ces exigences en lignes directrices de conception, puis en logiciels et solutions matérielles. Les vues des concepteurs concernant l'ouverture et la sécurité de la caméra ainsi que ce que l'utilisateur peut et est autorisé à faire sont intégrées, ou plutôt, matérialisées dans la caméra portée. Cependant, la demande des responsables politiques que la caméra enregistre les actes de violence à l'encontre des agents de police et les troubles commis dans l'espace public n'est pas entièrement satisfaite dans la mesure où il existe une différence cruciale entre l'enregistrement systématique et continu et l'enregistrement basé sur un incident [HAM 10]. Dans le premier cas, la responsabilité de fournir des images de qualité incombe en partie à l'objet (enregistrement, stockage et/ou envoi automatique) et en partie à l'utilisateur (orientation correcte de la caméra). Cet enregistrement automatique implique également une surveillance constante des actions des policiers, ce qui conduit à une plus grande objectivité mais aussi, comme nous le verrons, à une plus grande réticence des agents, qui se sentent alors surveillés, à utiliser cette caméra et/ou à une plus grande méfiance entre collègues. Le deuxième cas, qui est la configuration choisie pour la Zepcam, délègue la majeure responsabilité de ce qui est enregistré à l'utilisateur (c'est lui qui déclenche l'enregistrement), ce qui conduit à des enregistrements plus sélectifs, potentiellement biaisés et secrets — même si la caméra est équipée d'une mémoire tampon de sorte qu'elle enregistre ce qui s'est passé 10 secondes avant le déclenchement de l'enregistrement.

La conception de cette caméra portée par la police sur le modèle d'une caméra de sport extrême existante n'est pas sans conséquences quant aux objectifs et fonctionnalités qui peuvent être réalisés ou matérialisés. Recourir à un produit existant et y bricoler de nouvelles fonctions conduit certes à une caméra robuste qui peut être utilisée dans toutes sortes de conditions météorologiques, mais cela contraint la façon dont les sujets surveillés peuvent être notifiés de la présence et de l'enregistrement de la caméra ainsi que le traitement ultérieur des séquences filmées. En partant d'une caméra existante, ces éléments n'ont pas été considérés comme fondamentaux dans la conception et donc comme nécessitant, éventuellement, un nouveau design de la caméra portée. Le boîtier contenant l'objectif de la caméra tout comme le bracelet-télécommande sont noirs et difficilement perceptibles la nuit tandis que c'est à partir des prérequis fournis par les responsables politiques, et non à partir de la pratique des agents de police, que la délégation des responsabilités a été effectuée entre les différents acteurs humains et non-humains.

\section{Les caméras portées en action : entre résistances et nouveaux usages}

Ce parcours biographique de la Zepcam montre que ces caméras présentent une certaine ambiguïté et un large éventail d'objectifs potentiels. Toutefois, la phase de conception ne saurait marquer la fin de cette biographie, ce à double titre. Outre que, dans le cadre de la Zepcam, ce sont les utilisateurs les agents de police - qui se doivent de naviguer dans et résoudre les ambiguïtés et possible confusion liées aux objectifs et design de la caméra, délimiter le parcours biographique de la Zepcam aux choix des responsables politiques et des concepteurs risque de renforcer, même de façon implicite, ce que Rogers appelle le «biais pro-innovation », à savoir la vue qu' " une innovation devrait être diffusée et adoptée par tous les membres d'un système social » [ROG 03, p. 25]. Or, l'utilisation et l'appropriation de la caméra portée présentent des résultats très mitigés, interrogeant, ce faisant, l'interprétation de cet objet comme une innovation. 


\subsection{Un utilisateur configuré en décalage l'utilisateur réel}

Pour utiliser la caméra, les policiers doivent suivre une série d'étapes qui sont expliquées par Zepcam dans un manuel fourni aux utilisateurs. La caméra doit être connectée au câble qui la relie au boîtier d'interface-utilisateur ; la télécommande quant à elle peut être portée au poignet. En vertu de la loi, la caméra doit également être portée de façon à être vue et reconnue comme telle ou sa présence doit être notifiée aux éventuels sujets surveillés.

Au cours de nos observations, nous avons remarqué que connecter la caméra au boîtier d'interface était difficile, surtout lorsqu'il fallait vérifier en même temps l'angle de la caméra :

«Voyons si la caméra est correctement orientée [ouverture de la veste, sortie du boîtier, connexion, vérification, verrouillage de l'appareil et retour dans la veste] »(Observations, Enschede, 10 novembre 2012).

Comme le boîtier est portée à l'intérieur de la veste d'uniforme, ajuster la caméra et vérifier l'angle de prise de vue pendant une ronde signifie «se déshabiller», ce qui est jugé comme non professionnel par les policiers en service (observations, Enschede, 10 novembre 2012). De même, bien que la télécommande portée au poignet permette de déclencher un enregistrement en appuyant sur le bouton dédié, donc sans avoir à sortir le boîtier d'interface, celle-ci est également portée, en pratique, sous les manches de la veste. Ainsi, la LED qui indique que la caméra est ou non en marche n'est pas directement visible par ses utilisateurs (et encore moins par les acteurs impliqués), ce qui est source d'incertitude et de confusion quant à l'activité de la caméra. Comme nous l'ont signalé les policiers lors de nos observations, résoudre cette incertitude requerrait, ici aussi, de sortir le boîtier de la poche interne de l'uniforme (afin d'obtenir un retour visuel sur l'état de la caméra et son orientation), et donc de se dévêtir.

En outre, comme le regrette un policier :

«Regarder les images en direct devrait être possible [...] vous devez être capable de contrôler ce que vous filmez et quand » (Policier 1).

Aucun retour (feedback) afin, par exemple, de réorienter l'objectif si celui-ci est hors champ, n'est en effet possible sans sortir le boîtier d'interface-utilisateur. Aussi, quand bien même les agents de police ont la possibilité de regarder les images filmées lors de leur ronde et de taguer les séquences pertinentes, parce que cela leur demande encore une fois de «se déhabiller», le visionnage des séquences a lieu après une ronde de surveillance. Il est à noter que l'absence d'écran tactile pour faire défiler et taguer les images est considérée comme une contrainte additionnelle. Dans la mesure où les objectifs et fonctionnalités de la Zepcam ont été décidés par les responsables politiques et les concepteurs sans consulter les agents de police sur leurs pratiques et comportements jugés appropriés lorsqu'ils sont en service, l'usabilité de la caméra est limitée. En l'état, la caméra demande une attention - et constitue un poids - supplémentaire pendant les rondes, ce qui est en tension voire en contradiction avec la nécessité d'agir rapidement lors d'une intervention.

Ce travail additionnel se traduit-il par plus de sécurité ou une plus grande prévention de la violence à l'encontre des policiers, qui est l'objectif principal de la caméra pour les responsables politiques ? Comme l'indique un policier-utilisateur :

« Non, cela n'aide pas à réduire la violence à l'encontre des policiers. Ça reste une combinaison d'être humain et de caméra, et il est difficile de prouver qu'il y a eu moins d'incidents contre le personnel de police dus à la caméra » (Policier 2).

Non seulement la caméra portée ne permettrait pas de réduire les violences envers les agents de police, mais elle peut elle-même être source de danger. Lors de nos observations, un policier a fait remarquer à l'un de ses collègues que ce dernier avait mal positionné la caméra : 
« Tu ne la portes pas correctement : tu dois cacher le câble... Ça peut être dangereux ! Tu dois t'assurer que le câble est bien sous ta veste »(Observations, Enschede, 10 novembre 2012).

Si l'objectif de protection des agents de police demande que le port de la caméra ne présente pas de risque ou ne constitue pas un danger pour eux une fois dans l'espace public, il requiert cependant un soin particulier de la part des utilisateurs : ceux-ci doivent veiller à ce que le câble reliant la caméra au boîtier d'interface soit dissimulé sous leurs vêtements. En fait, les premières utilisations de la Zepcam par les policiers ont montré que le câble de connexion pouvait être tiré et utilisé contre l'agent (comme câble d'étranglement). Initialement fixe, il a été modifié : rendu séparable en son milieu, il se casse en deux s'il est tiré. Malgré ce changement, la caméra et son câble demandent toujours une attention conséquente de la part des policiers.

Par ailleurs, l'utilisation de la caméra, et surtout de ses images en tant que preuves, nécessite une organisation informatique particulière, jugée très contraignante. Différents protocoles ont été observés et rapportés lors de nos observations et entretiens. À Rotterdam, toutes les images enregistrées sont téléchargées sur un ordinateur autonome par un agent spécifiquement autorisé à le faire. Les séquences qui sont marquées (tagged) ou estimées devant être gardées sont conservées pour examen. Le reste est supprimé. À Enschede, seules les images jugées importantes sont téléchargées sur un ordinateur dédié qui n'est pas connecté à un réseau. Les images sont ensuite examinées par l'utilisateur de la caméra avec un officier supérieur, puis stockées dans un dossier local ou supprimées. Une fois par mois, celles qui ne sont plus nécessaires sont effacées. Cependant, comme l'indique un utilisateur de la caméra, la difficulté d'obtenir et d'accéder aux images sur son propre ordinateur est un inconvénient :

«Il devrait être plus facile de vider cette chose... d'en sortir les séquences, de les avoir prêtes à être visionnées dès que vous êtes sur votre propre PC » (Policier 3 ).

Cette rigidité s'explique par le fait que les séquences ne peuvent être utilisées comme preuves officielles que si elles sont correctement sécurisées : cette exigence des responsables politiques a été traduite par la création d'un système garantissant que les données ne peuvent être téléchargées que sur un ordinateur dédié par un utilisateur spécifique.

En décalage avec les pratiques des agents de police, la caméra portée est difficilement appropriée et intégrée à leurs usages. C'est en général lors des briefings qui ont lieu avant une ronde de surveillance que les caméras sont vérifiées et réparties entre les différentes équipes :

« Eh bien, vous voyez [le policier essaie de nous montrer des images de la nuit précédente], oh... celle-ci ne fonctionne pas. [À un collègue :] On en a une autre ? Non ? Ah, eh bien, alors pas de caméra cette nuit »(Observations, Enschede, 3 octobre 2012).

Bien que cette équipe se soit finalement vue attribuer une caméra, elle n'a pas pris le temps d'essayer de comprendre ni de résoudre pourquoi la caméra qui lui était assignée ne fonctionnait pas, ni même de la remplacer par un appareil en état de marche. Comparée aux autres équipements que les agents doivent porter, la Zepcam est traitée comme un élément accessoire et de dernière minute.

\subsection{Des objectifs ambivalents redéfinis par les utilisateurs}

Outre qu'elles sont utilisées de façon restreinte, lorsqu'elles le sont, les caméras portées donnent lieu à des usages dont les finalités sont en tension avec les objectifs définis par les responsables politiques et inscrits par les concepteurs.

La Zepcam joue un rôle ambivalent pour les policiers, lequel est lié à la relative (in-)visibilité de la caméra. En écho aux discussions sur la vidéosurveillance et sa fonction préventive [BRA 13], la condition préalable à la prévention (des violences) est que les citoyens reconnaissent la caméra portée 
comme, effectivement, une caméra. Lorsque sa présence est notifiée, le plus souvent oralement, elle peut avoir un rôle préventif :

«Quand on dit qu'ils sont filmés, ils deviennent plus silencieux » (Observations, Enschede, 3 octobre 2012).

Si tant est qu'elle est rendue visible, la caméra peut donc être utilisée intentionnellement afin de limiter les actes de violence à l'encontre des agents ${ }^{4}$. Néanmoins, comme le souligne un policier,

«C'est utile si les gens ne savent pas qu'ils sont filmés [...] Parfois, vous voulez enregistrer en secret, parfois non. Dans les quartiers animés la nuit, nous indiquons clairement que nous enregistrons » (Policier 2).

Dans la mesure où la responsabilité de l'enregistrement est déléguée à l'utilisateur et en l'absence de fonction unique et univoque inscrite dans la caméra, l'usage de celle-ci à couvert ou découvert est laissé à la discrétion de l'agent de police. D'ailleurs, comme l'indique un policier :

«Souvent les gens pensent que cette caméra est un microphone » (Observations, Enschede, 3 octobre 2012).

Non reconnaissable comme telle, la caméra permet d'enregistrer en secret. Elle peut donc être utilisée comme caméra de surveillance, afin de collecter des preuves et/ou d'identifier des individus, sans que les citoyens soient informés de leur enregistrement. Certes cette surveillance n'est pas constante ni continue, les enregistrements réalisées pendant une ronde ne constituant que quelques minutes de film, mais cet usage de la caméra sort du cadre légal et prévu par les responsables politiques.

À cet égard, l'un des policiers rencontrés, qui doute que la caméra portée permette de remplir sa visée première, à savoir réduire la violence à l'encontre des agents de police, signale qu'

«Elle est cependant très pratique pour la collecte de preuves [...] Nous avons beaucoup de cas résolus en utilisant les images de la caméra » (Policier 2).

Lorsqu'elles enregistrent une infraction et/ou un suspect, les images fournies par la caméra peuvent appuyer les policiers dans leurs enquêtes. Les images sont ainsi considérées comme une source fiable de données. L'inscription réalisée par les concepteurs de la Zepcam est, ici, un succès. Ses images étant également mobilisées par les policiers pour vérifier la justesse du rapport qu'ils rédigent à la suite de leur ronde nocturne, la caméra est perçue comme un «témoin objectif ». Au cours d'un entretien, un policier a notamment évoqué que le taux d'adrénaline des agents de police en intervention entraîne une certaine étroitesse de vue qui peut bloquer leur «vision objective ». Dans ce contexte, la caméra portée permet de clarifier, a posteriori, ce qui s'est réellement passé :

«Vous tapez votre rapport, et après, vous avez accès aux images de la nuit avec la caméra » (Policier 1).

Certains utilisateurs déclarent qu'il est en effet utile de clarifier certains détails et de réévaluer les situations avec du recul. Toutefois, les images ne sont pas uniquement utilisées après une ronde mais aussi avant celle-ci.

\footnotetext{
${ }^{4}$ Aucun lien direct n'a toutefois été rapporté entre l'utilisation de la caméra portée et la diminution de la violence à l'encontre des policiers [HAM 10].
} 
Intégrées à l'infrastructure informatique locale, les images issues de la caméra peuvent circuler, s'éloignant ainsi de la promesse de les conserver dans un lieu central. Cela conduit à des formes d'utilisation non envisagées ni souhaitées par les responsables politiques :

« Nous utilisons ces images pour réaliser des captures d'écran, que nous regardons à nouveau lors des briefings organisés avant les rondes de surveillance » (observations, Enschede, 3 octobre 2012)

Comme le révèle ce policier, la caméra et ses images peuvent être utilisées à des fins de profilage, c'est-à-dire afin d'identifier les «personnes à surveiller » lors de la ronde à venir. Ainsi, c'est avant et après la ronde de surveillance, plutôt que pendant celle-ci, et du fait de finalités non prévues par les responsables politiques et les concepteurs, que la caméra est manifestement utile pour ses utilisateurs.

Cette dernière n'a toutefois pas été perçue et reçue favorablement par tous les agents de police. Comme le souligne l'un des responsables politiques lui-même agent de police :

«Alors oui, certains collègues étaient réticents à utiliser la caméra, d'autres non. Des collègues enthousiastes, des collègues moins enthousiastes » (Responsable Rotterdam).

Si les attentes des policiers diffèrent quant à ce que la caméra peut faire et comment elle peut les aider lorsqu'ils sont en service, c'est aussi vis-à-vis de la fonction de «témoin objectif » de la caméra portée que les réticences se font le plus sentir : celle-ci peut constituer un outil de surveillance non seulement pour les citoyens mais aussi pour les policiers eux-mêmes. Cette forme d'auto-surveillance a d'ailleurs fait l'objet de discussions entre agents au cours d'une ronde, leur réticence à utiliser la caméra étant en partie due à un manque de clarté sur le contrôle des temps d'enregistrement et des séquences filmées. Dans la mesure où le script de la caméra autorise différentes configurations de contrôle d'accès aux images, des préoccupations ont été exprimées concernant l'emploi de la caméra : plutôt que de protéger les agents de police ou de justifier leurs interventions, celle-ci pourrait servir à collecter des éléments de preuve qui pourraient être utilisés contre eux. Les différentes fonctions et objectifs de la caméra, tels qu'ils ont été énoncés par les responsables et traduits par les concepteurs sont ainsi source d'ambiguité et de méfiance.

Les caméras portées et leurs images revêtent un caractère expérimental dans les pratiques de travail de la police. Dans les cas observés, elles oscillent entre dispositifs de surveillance et dispositifs de profilage, deux fonctions éloignées des objectifs visés par les responsables politiques. Des cas de glissement ou de détournement de fonction apparaissent donc dans les assemblages surveillants locaux. Du fait de ses fonctionnalités équivoques et de son décalage avec les pratiques et usages des agents de police, qui découlent de leur non-implication dans la conception de la caméra portée, celle-ci fait l'objet de résistance et d'une appropriation limitée.

\section{Conclusion}

Cet article, centré sur le processus de développement et d'introduction d'un nouveau dispositif de surveillance dans le paysage nocturne néerlandais, montre l'imbrication de différentes échelles, acteurs et objectifs dans la biographie de la caméra portée. C'est en retraçant les différentes traductions et inscriptions qui ont pris place dans la définition, la conception et l'utilisation de la Zepcam que nous avons construit cette biographie. Parce qu'elle montre que le sens d'un objet technologique n'est pas unique ni figé mais qu'il se transforme selon les groupes qui s'en saisissent, celle-ci contribue au façonnement d'une «culture technique » utile aux responsables politiques, concepteurs et utilisateurs.

Le but de la caméra est déjà multiple lorsque les responsables politiques dressent une liste des demandes «fonctionnelles» qu'elle doit remplir. S'appuyant sur une combinaison de lois de surveillance et de police existantes, l'objet proposé sur le papier doit être à la fois un outil de prévention de la violence à l'encontre de policiers et une caméra de surveillance mobile. C'est en ce 
sens que les responsables «inscrivent » les concepteurs : ils leur fournissent un script que l'objet doit matérialiser et c'est sur la base d'une caméra de sports extrêmes que ces demandes ont été traduites par les concepteurs. Toute traduction implique toutefois une réappropriation. Dans ce processus qui n'est ni direct ni linéaire, non seulement le choix de modifier une technologie existante influence les options technologiques possibles dans le processus de développement, mais, ce faisant, il néglige aussi l'utilisateur final et le contexte d'utilisation de la future caméra. Ainsi, les concepteurs ont mis l'accent sur la sécurité des séquences vidéo et le contrôle d'accès à ces dernières, déléguant des responsabilités à l'utilisateur et l'appareil, mais ne résolvant pas certaines ambiguïtés de la caméra. C'est dans les pratiques des agents de police que se matérialisent ce que fait et est la caméra. Celle-ci semble être surtout utilisée comme une caméra de vidéosurveillance mobile et comme un témoin objectif secret, plutôt que comme un dispositif de protection contre les actes de violence dirigés contre la police. En contraste avec les représentations auxquelles elle a donné lieu, en particulier dans les pays anglosaxons, la caméra en tant que «témoin objectif» ne vient pas surveiller la police (même si c'est une crainte des agents), afin de contrôler la légitimité et la légalité de ses pratiques [LIP 16], mais elle lui permet plutôt de vérifier les décisions prises en interne et d'identifier de potentiels fauteurs de troubles. Ce faisant, elle participe à la transformation des modes de surveillance, laquelle n'est plus fixe et limitée à des espaces restreints dans l'espace public mais mobile et permettant un contrôle social déterritorialisé.

\section{Bibliographie}

[AKR 90] AKRICH, M., « De la sociologie des techniques à une sociologie des usages : L'impossible intégration du magnétoscope dans les réseaux câbles de première génération », Techniques et culture, n¹6, p. 83-110, 1990.

[AKR 92] AKRICH, M., «The De-scription of Technical Objects », dans W. BIJKER et J. LAW, J. (dir.), Shaping Technology / Building Society : Studies in Sociotechnical Change, Cambridge, The MIT Press, p. $205-224,1992$.

[AKR 95] AKRICH, M., «User Representations : Practices, Methods and Sociology », dans A. RIP, T. MISA et J. SCHOT (dir.) Managing Technology in Society: The Approach of Constructive Technology Assessment, Londres, Pinter, p. 167-184, 1995.

[BIJ 92] BIJKER, W. et LAW, J. (dir) Shaping Technology / Building Society : Studies in Sociotechnical Change, Cambridge, The MIT Press, 1992.

[BRA 13] BRAnds, J., Schwanen, T. et VAn Aalst, I., « What Are You Looking At ? Visitors' Perspectives on CCTV in the Night-Time Economy », European Urban and Regional Studies, vol. 23, n 1, p. 23-39, 2013.

[CHO 17] ChOuteau, M., Forest, J. et NGuYen, C., «Quand la culture d'innovation fait écran à la culture technique », Technologie et innovation, vol. 17, $\mathrm{n}^{\circ} 2$, p. 21-40, 2017.

[CLA 93] Clarke, A. et Montini, T., « The Many Faces of RU 486 : Tales of Situated Knowledges and Technological Contestations », Science, Technology \& Human Values, vol. 18, n 1, p. 42-78, 1993.

[HAM 10] HAM, T., FERWEDA, H. et KuPPENS, J., Cameratoezicht Beweging. Ervaringen met nieuwe vormen van cameratoezicht bij de Nederlandse politie, Arnhem, Buro Beke, 2010.

[HOM 03] Homburg, G. et DeKKers, S., Cameratoezicht in de openbare ruimte, La Haye, College bescherming persoonsgegevens, 2003.

[JAN 09] JANSEN, D. R. (2009). Juridisch Kader MBT Inzet Bodycams, Rapport de recherche de la police néerlandaise.

[LIP 16] LIPPERT, R. et NEWELl, B., «The Privacy and Surveillance Implications of Police Body Cameras », Surveillance \& Society, vol. 14, $\mathrm{n}^{\circ}$ 1, p. 113-116, 2016.

[LYO 05] LYON, D., «The Border is Everywhere : ID Cards, Surveillance and the Other », dans E. ZUREIK et M. B. SALTER (dir.), Global Surveillance and Policing : Borders, Security, Identity, Cullompton, Willan Publishing, p. 66$82,2015$.

[OUD 04] Oudshoorn, N., Rommes, E. et StIEnSTRA, M., « Configuring the User as Everybody : Gender and Design Cultures in Information and Communication Technologies », Science, Technology \& Human Values, vol. 29, $\mathrm{n}^{\circ} 1$, p. 30-63, 2004. 
[PIN 12] PINCH, T. et BIJKER, W., « The Social Construction of Facts and Artifacts », dans W. BIJKER, T. HUGHES et T. PINCH (dir.), The Social Construction of Technological Systems: New Direction in the Sociology and History of Technology, Cambridge, The MIT Press, p. 11-44, 2012.

[ROG 03] ROGERS, E., Diffusion of Innovation, New York, Free Press, 2003.

[SCH 15] SCHNEIDER, C., « Police Image Work in an Era of Social Media: YouTube and the 2007 Montebello Summit Protest», dans D. TrotTIER et C. Fuchs (dir.), Social Media, Politics and the State : Protests, Revolution, Riots, Crime and Policing in an Age of Facebook, Twitter and YouTube, Londres, Routledge, p. 227-245, 2015.

[TIM 17] TIMAN, T., «Policy, design and use of police-worn bodycameras in the Netherlands », dans S. ADAMS, N. PURTOVA et R. LEENES (dir.), Under Observation: The Interplay between eHealth and Surveillance, Dordrecht, Springer, p.139-162, 2017.

[TYL 04] TYLER, T., «Enhancing Police Legitimacy », Annals of the American Academy of Political and Social Science, vol. 593, nº 1, p. 84-99, 2004.

[WOO 90] WoolgaR, S., «Configuring the User: The Case of Usability Trials », The Sociological Review, vol. 38, $\mathrm{n}^{\circ}$ S1, p. 58-99, 1990. 